\title{
As borboletas (Lepidoptera, Papilionoidea) do Campus Universitário Darcy Ribeiro (Distrito Federal, Brasil)
}

\author{
Carlos Eduardo Guimarães Pinheiro ${ }^{1,3}$, Ivan Constantinov Malinov ${ }^{1}$, Thiago Oliveira Andrade ${ }^{1}$,
}

Jonas Brochado Maravalhas ${ }^{1}$, Marcelo Brito Moussallem de Andrade ${ }^{1}$, Luis Paulo Aguiar de Deus ${ }^{1}$,

Luiz Gustavo Perrut Pedrosa ${ }^{2}$ \& Gabriel Vargas Zanatta ${ }^{2}$

${ }^{1}$ Departamento de Zoologia, Instituto de Biologia, Universidade de Brasília - UnB, CEP 70910-900, Brasília, DF, Brasil, e-mail: cegp@unb.br

${ }^{2}$ Departamento de Engenharia Florestal, Faculdade de Tecnologia,

Universidade de Brasília - UnB,

CEP 70910-900, Brasília, DF, Brasil

${ }^{3}$ Autor para correspondência: Carlos Eduardo Guimarães Pinheiro,e-mail: cegp@unb.br

PINHEIRO, C.E.G., MALINOV, I.C., ANDRADE, T.O., MARAVALHAS, J., MOUSSALLEM, M., DEUS, L.P.A., PEDROSA, L.G.P. \& ZANATTA, G. 2008. The butterflies (Lepidoptera, Papilionoidea) of the University Campus Darcy Ribeiro (Distrito Federal, Brasil). Biota Neotrop. 8(4): http://www.biotaneotropica.org.br/ v8n4/en/abstract?inventory+bn00608042008.

\begin{abstract}
The Brazilian cerrado, the second largest bioma in this country, is now constituted only by fragments of vegetation that together correspond to less than $20 \%$ of its original vegetation. This study investigates the butterfly fauna found in fragments of cerrado sensu stricto and gallery forest of the University Campus Darcy Ribeiro. A list containing 128 butterfly species, corresponding to approximately $25 \%$ of all Papilionoidea found in the Distrito Federal is presented. Some factors affecting the species richness of butterflies in the study sites are also discussed.
\end{abstract}

Keywords: cerrado vegetation, conservation, habitat fragmentation, inventory.

PINHEIRO, C.E.G., MALINOV, I.C., ANDRADE, T.O., MARAVALHAS, J., MOUSSALLEM, M., DEUS, L.P.A., PEDROSA, L.G.P. \& ZANATTA, G. 2008. As borboletas (Lepidoptera, Papilionoidea) do Campus Universitário Darcy Ribeiro (Distrito Federal, Brasil). Biota Neotrop. 8(4): http://www.biotaneotropica.org. br/v8n4/pt/abstract?inventory+bn00608042008.

Resumo: O cerrado brasileiro, considerado o segundo maior bioma do país em extensão territorial, encontra-se atualmente constituído apenas por fragmentos de vegetação que em conjunto representam menos de $20 \%$ de sua vegetação original. Neste trabalho nós investigamos a fauna remanescente de borboletas em fragmentos de cerrado sensu stricto e mata ciliar do campus universitário Darcy Ribeiro. No total foram encontradas 128 espécies correspondendo a aproximadamente $25 \%$ da fauna de borboletas do Distrito Federal. Alguns fatores que afetam a riqueza de espécies de borboletas nas áreas de estudo são também discutidos.

Palavras-chave: cerrado, conservação, fragmentação de habitat, inventário. 


\section{Introdução}

O cerrado é atualmente reconhecido como um dos biomas terrestres com maior diversidade de animais de todo o planeta, incluindo lepidópteros (Brown Jr. \& Mielke 1967a, b, Brown Jr. \& Gifford 2002). Apesar de toda essa riqueza o cerrado vem sofrendo um intenso processo de destruição e transformação de sua paisagem natural devido à urbanização e implantação de atividades agrícolas - geralmente envolvendo grandes monoculturas, criação de gado e a produção de carvão, restando na atualidade apenas fragmentos ou ilhas de vegetação equivalentes a menos de $20 \%$ da área originalmente ocupada (Myers et al. 2000). Estudos recentes mostram que a fragmentação de um bioma pode levar a perda em biodiversidade, especialmente no caso de invertebrados (DeVries et al. 1999, Hill et al. 2001, Lewinsohn et al. 2005, Pacheco \& Vasconcelos 2007). Sendo assim, é necessário voltar a nossa atenção para esses fragmentos de vegetação, pois além de representarem tudo o que restou de um determinado bioma, também constituem a única esperança para a conservação da fauna ainda existente e para qualquer tentativa que vise à recuperação da vegetação original em determinadas regiões. Neste trabalho nós investigamos a fauna de borboletas (Papilionoidea) encontrada em fragmentos de vegetação de cerrado localizados no campus universitário Darcy Ribeiro da Universidade de Brasília.

Inaugurado em 21 de abril de 1962 o campus ocupa uma área de $3.950 .579 \mathrm{~m}^{2}$ na Asa Norte de Brasília, junto às margens da Represa

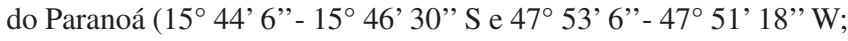
750-800 m de altitude), apresentando uma localização intermediária entre algumas das unidades de conservação mais importantes do Brasil central, como o Parque Nacional de Brasília, a Área de Proteção Ambiental do Gama e Cabeça de Veado e a Estação Ecológica de Águas Emendadas. Desde a sua inauguração a área do campus vem sofrendo um intenso processo de transformação de suas paisagens naturais. Entretanto, pouco se tem feito para a conservação da vegetação nativa e da fauna dessa área, restando hoje apenas algumas manchas de cerrado sensu stricto - o tipo de vegetação predominante no local (veja Goodland 1971, Eiten 1972, Oliveira-Filho \& Ratter 2002 para uma descrição dos tipos fisionômicos de vegetação de cerrado) e pequenas manchas de matas ciliares próximas a nascentes. Uma tentativa para se conservar a fauna e flora da região foi a criação de uma reserva na área do Centro Esportivo Universitátio, atualmente conhecida como Reserva do Centro Olímpico. Entretanto, pouco se fez pela implantação efetiva dessa reserva que tem estado sempre sujeita à presença de plantas invasoras, animais domésticos, residentes clandestinos e depósitos de lixo, além de sofrer anualmente a ação de incêndios de grandes proporções.

Apesar de todos esses problemas alguns estudos de fauna desenvolvidos no campus nos últimos anos têm mostrado que esses fragmentos de vegetação podem ainda suportar uma grande riqueza de espécies. M. Bagno (com. pess.) observou aproximadamente 180 espécies de aves na região do campus, correspondendo a aproximadamente $40 \%$ de todas as aves do Distrito Federal, sugerindo que os fragmentos de vegetação ainda existentes podem desempenhar um papel bastante relevante na conservação da fauna local. Neste trabalho nós apresentamos uma listagem das borboletas (Papilionoidea) do campus. Nossos objetivos são: 1) conhecer a fauna de borboletas do campus e permitir o desenvolvimento de diferentes tipos de pesquisas científicas envolvendo a mesma; 2) permitir que a diversidade de espécies encontrada possa ser monitorada em estudos futuros; e 3) incentivar a criação e a manutenção de reservas e áreas de conservação nos fragmentos de vegetação de cerrado ainda existentes.

\section{Material e Métodos}

As localidades amostradas no campus incluem a Reserva do Centro Olímpico (RCO) que além de cerrado sensu stricto (aprox. 8 ha), contém ainda uma área de mata ciliar (2 ha) e várias áreas originalmente ocupadas por vegetação de cerrado e atualmente apresentando diferentes estágios de sucessão ecológica, a Estação Experimental de Biologia (EEB), que contém uma área de cerrado sensu stricto (3 ha) e uma pequena área de mata junto à Represa do Paranoá, e a região do arboreto, que também inclui áreas de cerrado sensu stricto ( $2 \mathrm{ha}$ ) e uma pequena mata ciliar ( $0.5 \mathrm{ha}$ ) ao longo de um córrego cuja nascente se encontra no Parque Olhos D'Água, em área adjacente ao campus (Figura 1).

As amostragens da fauna de borboletas foram realizadas mensalmente por Pinheiro em 2004 e 2005 na RCO e outras localidades do campus; por Pinheiro, Malinov e Oliveira em 2006 e 2007 na RCO (amostragens semanais); e por Pinheiro e os demais autores deste trabalho em janeiro de 2008 na EEB, RCO e na região do Arboreto (amostragens diárias). Essas amostragens foram realizadas com redes entomológicas e armadilhas contendo iscas de banana fermentada em caldo de cana (uma ilustração desse tipo de armadilha pode ser encontrada em DeVries 1987). Todos os espécimes coletados foram incluídos na Coleção Entomológica da Universidade de Brasília. Espécies com distribuições geográficas presumidas ou encontradas apenas em regiões vizinhas à área do campus não foram incluídas na nossa listagem. A classificação adotada segue Lamas (2004).

\section{Resultados e Discussão}

Uma lista das borboletas (Papilionoidea) encontradas no campus universitário Darcy Ribeiro com suas respectivas classificações e habitats em que foram observadas ou capturadas é apresentada na Tabela 1. No total foram obtidas 128 espécies, correspondendo a aproximadamente $25 \%$ de todos os Papilionoidea encontrados no Distrito Federal (Emery et al. 2006). Estes valores mostram claramente que os fragmentos de vegetação existentes no campus ainda contém uma riqueza de espécies de borboletas relativamente alta.

Devido à predominância da vegetação de cerrado sensu stricto nestes fragmentos, a maior parte das espécies encontradas ( $\mathrm{n}=68 \mathrm{ou}$

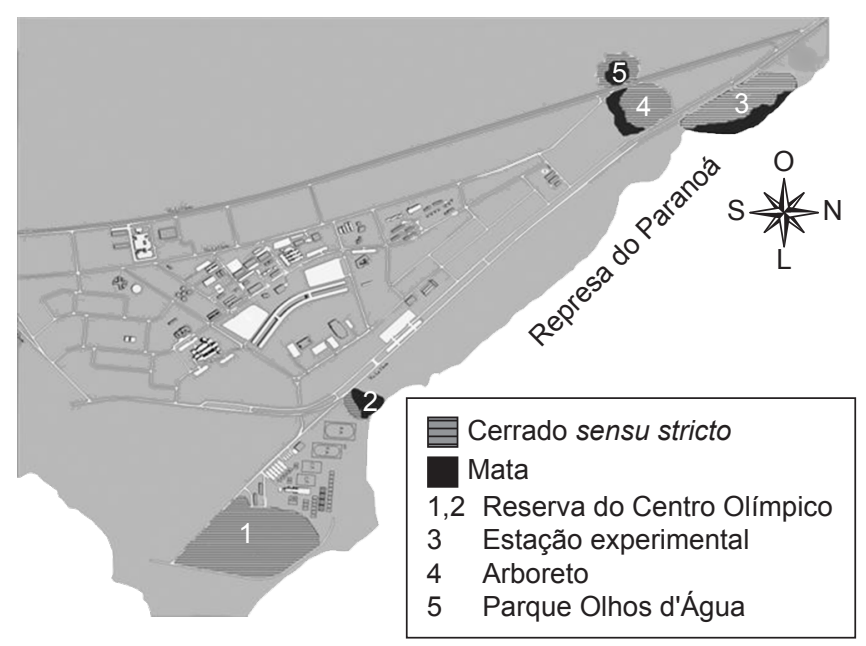

Figura 1. Localização das áreas de cerrado sensu stricto e mata ciliar no Campus Universitário Darcy Ribeiro, Brasília, Distrito Federal.

Figure 1. Locations of cerrado sensu stricto and gallery forest areas in the University Campus Darcy Ribeiro, Brasília, Distrito Federal. 
Tabela 1. Lista das borboletas (Papilionoidea) do Campus Universitário Darcy Ribeiro, Brasília, Distrito Federal. Habitats incluem: CSS (cerrado sensu stricto), MC (mata ciliar), AS (área em sucessão) e J (jardins).

Table 1. List of butterflies (Papilionoidea) of the University Campus Darcy Ribeiro, Brasília, Distrito Federal. Habitats include: CSS (cerrado sensu stricto), MC (gallery forest), AS (successional fields) e J (gardens).

\begin{tabular}{|c|c|c|}
\hline & Família/subfamília/tribo/espécies & Habitats \\
\hline \multicolumn{3}{|c|}{ PAPILIONIDAE } \\
\hline \multicolumn{3}{|c|}{ Papilioninae - Troidini } \\
\hline 1 & Battus crassus crassus (Cramer, 1777) & MC \\
\hline 2 & $\begin{array}{l}\text { Battus polydamas polydamas (Linnaeus, } \\
1758 \text { ) }\end{array}$ & CSS, MC, AS J \\
\hline \multirow[t]{2}{*}{3} & $\begin{array}{l}\text { Parides anchises foetterlei (Rothschild \& } \\
\text { Jordan, 1906) }\end{array}$ & $\mathrm{MC}$ \\
\hline & Papilioninae - Papilionini & \\
\hline 4 & $\begin{array}{l}\text { Heraclides anchisiades capys (Hübner, } \\
\text { [1809]) }\end{array}$ & $\mathrm{CSS}, \mathrm{MC}, \mathrm{AS}, \mathrm{J}$ \\
\hline 5 & $\begin{array}{l}\text { Heraclides thoas brasiliensis (Rothschild } \\
\text { \& Jordan, 1906) }\end{array}$ & $\mathrm{CSS}, \mathrm{MC}, \mathrm{AS}, \mathrm{J}$ \\
\hline \multicolumn{3}{|c|}{ PIERIDAE } \\
\hline \multicolumn{3}{|c|}{ Coliadinae } \\
\hline 6 & Anteos clorinde (Godart, [1824]) & CSS \\
\hline 7 & Anteos menippe (Hübner, [1818]) & CSS \\
\hline 8 & $\begin{array}{l}\text { Phoebis argante argante } \\
\text { (Fabricius, 1775) }\end{array}$ & CSS, AS \\
\hline 9 & Phoebis philea philea (Linnaeus, 1763) & CSS, AS \\
\hline 10 & Phoebis sennae sennae (Linnaeus, 1758) & $\mathrm{CSS}, \mathrm{AS}, \mathrm{J}$ \\
\hline 11 & Aphrissa statira statira (Cramer, 1777) & CSS, AS, J \\
\hline 12 & Pyrisitia leuce leuce (Boisduval, 1836) & CSS, AS \\
\hline 13 & Pyrisitia nise tenella (Boisduval, 1836) & AS \\
\hline 14 & Eurema albula albula (Cramer, 1775) & CSS, AS, J \\
\hline 15 & Eurema deva doris (Röber, 1909) & CSS, AS \\
\hline 16 & Eurema elathea elathea (Cramer, 1777) & $\mathrm{CSS}, \mathrm{AS}, \mathrm{J}$ \\
\hline \multirow[t]{2}{*}{17} & Eurema phiale paula (Röber, 1909) & CSS, AS, J \\
\hline & Pierinae - Pierini & \\
\hline 18 & Appias drusilla drusilla (Cramer, 1777) & CSS, AS, J \\
\hline 19 & Ascia monuste orseis (Godart, 1819) & CSS, AS, J \\
\hline \multicolumn{3}{|c|}{ LYCAENIDAE } \\
\hline \multicolumn{3}{|c|}{ Polyommatinae } \\
\hline 20 & Leptotes cassius cassius (Cramer, 1775) & CSS, AS \\
\hline 21 & Hemiargus hanno (Stoll, 1790) & CSS \\
\hline \multirow[t]{2}{*}{22} & Zizula cyna (Edwards, 1881) & CSS \\
\hline & Theclinae - Eumaeini & \\
\hline 23 & Theritas triquetra (Hewitson, 1865) & CSS \\
\hline 24 & Arawacus ellida (Hewitson, 1867) & MC \\
\hline 25 & Cyanophrys herodotus (Fabricius, 1793) & CSS \\
\hline 26 & Bistonina mantica (Druce, 1907) & CSS \\
\hline 27 & Strymon mulucha (Hewitson, 1867) & CSS \\
\hline 28 & Tmolus echion (Linnaeus, 1767) & CSS \\
\hline 29 & Nicolaea socia (Hewitson, 1868) & CSS \\
\hline \multirow[t]{3}{*}{30} & Olynthus essus (Herrich-Schäffer, [1853]) & CSS \\
\hline & Riodinidae & \\
\hline & Euselasiinae - Euselasiini & \\
\hline \multirow[t]{2}{*}{31} & Euselasia mys cytis Stichel, 1919 & CSS \\
\hline & Riodininae - Riodinini & \\
\hline 32 & $\begin{array}{l}\text { Lyropteryx terpsichore terpsichore } \\
\text { Westwood, } 1851\end{array}$ & CSS \\
\hline
\end{tabular}

Tabela 1. Continuação...

\begin{tabular}{|c|c|c|}
\hline & Família/subfamília/tribo/espécies & Habitats \\
\hline 33 & $\begin{array}{l}\text { Lasaia agesilas agesilas (Latreille, 1809) } \\
\text { Riodininae - Symmachiini }\end{array}$ & CSS \\
\hline 34 & $\begin{array}{l}\text { Symmachia hippodice Godman, } 1903 \\
\text { Riodininae - Tribo Incertae Sedis }\end{array}$ & CSS \\
\hline 35 & $\begin{array}{l}\text { Apodemia paucipuncta Spitz, } 1930 \\
\text { Riodininae - Nymphidiini }\end{array}$ & CSS \\
\hline 36 & Ariconias glaphyra (Westwood, 1851) & CSS \\
\hline 37 & $\begin{array}{l}\text { Synargis calyce } \\
\text { (C. Felder \& R. Felder, 1862) }\end{array}$ & CSS \\
\hline 38 & Synargis agle (Hewitson, [1853]) & CSS \\
\hline 39 & Synargis gela (Hewitson, [1853]) & CSS \\
\hline 40 & $\begin{array}{l}\text { Nymphidium leucosia leucosia } \\
\text { (Hübner, [1806]) }\end{array}$ & CSS \\
\hline 41 & Nymphidium lisimon (Stoll, 1790) & $\mathrm{MC}$ \\
\hline 42 & Theope eudocia Westwwod, 1851 & $\mathrm{MC}$ \\
\hline 43 & $\begin{array}{l}\text { Theope pieridoides } \\
\text { C. Felder \& R. Felder, } 1865\end{array}$ & CSS \\
\hline 44 & $\begin{array}{l}\text { Theope terambus (Godart, [1824]) } \\
\text { Riodininae - Stalachtini }\end{array}$ & CSS \\
\hline 45 & $\begin{array}{l}\text { Stalachtis phlegia phlegetontia } \\
\text { (Perty, 1833) } \\
\text { NYMPHALIDAE } \\
\text { Libytheinae }\end{array}$ & $\mathrm{CSS}, \mathrm{MC}, \mathrm{J}$ \\
\hline 46 & $\begin{array}{l}\text { Libytheana carinenta (Cramer, 1779) } \\
\text { Danainae - Danaini }\end{array}$ & MC \\
\hline 47 & Danaus gilippus gilippus (Cramer, 1775) & $\mathrm{MC}, \mathrm{J}$ \\
\hline 48 & Danaus eresimus plexaure (Godart, 1819) & $\mathrm{MC}, \mathrm{J}$ \\
\hline 49 & Danaus erippus (Cramer, 1775) & $\mathrm{MC}, \mathrm{J}$ \\
\hline 50 & $\begin{array}{l}\text { Lycorea halia discreta Haensch, } 1909 \\
\text { Ithomiinae - Tithoreini }\end{array}$ & $\mathrm{MC}$ \\
\hline 51 & $\begin{array}{l}\text { Tithorea harmonia pseudethra } \\
\text { Butler, } 1873\end{array}$ & $\mathrm{MC}$ \\
\hline 52 & $\begin{array}{l}\text { Aeria elara elarina (Oberthür, 1879) } \\
\text { Ithomiinae - Mechanitini }\end{array}$ & MC \\
\hline 53 & Methona themisto (Hübner, 1818) & $\mathrm{MC}$ \\
\hline 54 & $\begin{array}{l}\text { Thyridia psidii hippodamia } \\
\text { (Fabricius, 1775) }\end{array}$ & MC \\
\hline 55 & $\begin{array}{l}\text { Mechanitis lysimnia lysimnia } \\
\text { (Fabricius, 1793) }\end{array}$ & $\mathrm{MC}$ \\
\hline 56 & $\begin{array}{l}\text { Mechanitis polymnia casabranca } \\
\text { Haensch, } 1905\end{array}$ & MC \\
\hline 57 & $\begin{array}{l}\text { Ithominae - Napeogenini } \\
\text { Hypothyris ninonia daeta } \\
\text { (Boisduval, 1836) }\end{array}$ & $\mathrm{MC}$ \\
\hline 58 & $\begin{array}{l}\text { Ithomiinae - Ithomiini } \\
\text { Ithomia agnosia agnosia } \\
\text { (Hewitson, [1855]) }\end{array}$ & MC \\
\hline 59 & $\begin{array}{l}\text { Placidina euryanassa } \\
\text { (C. Felder \& R. Felder, 1860) } \\
\text { Ithomiinae - Dircennini }\end{array}$ & MC \\
\hline 60 & Dircenna dero dero (Hübner, 1823) & $\mathrm{MC}$ \\
\hline 61 & $\begin{array}{l}\text { Episcada hymenaea centralis } \\
\text { (Brown \& Mielke 1970) }\end{array}$ & MC \\
\hline & Ithomiinae - Godyridini & \\
\hline
\end{tabular}


Tabela 1. Continuação...

\begin{tabular}{|c|c|c|}
\hline & Família/subfamília/tribo/espécies & Habitats \\
\hline 62 & Hypoleria lavinia consimilis Talbot, 1928 & $\mathrm{MC}$ \\
\hline 63 & $\begin{array}{l}\text { Hypoleria sarepta goiana } \\
\text { d'Almeida, } 1951\end{array}$ & $\mathrm{MC}$ \\
\hline 64 & Brevioleria seba emyra (Haensch, 1905) & MC \\
\hline 65 & $\begin{array}{l}\text { Mcclungia cymo salonina } \\
\text { (Hewitson, 1855) }\end{array}$ & $\mathrm{MC}$ \\
\hline 66 & Heterosais edessa (Hewitson, [1855]) & MC \\
\hline 67 & Pseudoscada erruca (Hewitson, 1855) & $\mathrm{MC}$ \\
\hline 68 & $\begin{array}{l}\text { Pseudoscada acilla quadrifasciata } \\
\text { Talbot, } 1928 \\
\text { Morphinae - Morphini }\end{array}$ & $\mathrm{MC}$ \\
\hline 69 & $\begin{array}{l}\text { Grasseia menelaus coeruleus } \\
\text { (Perry, 1810) }\end{array}$ & $\mathrm{MC}$ \\
\hline 70 & $\begin{array}{l}\text { Morpho helenor achillides } \\
\text { (C. Felder \& R. Felder, 1867) } \\
\text { Morphinae - Brassolini }\end{array}$ & $\mathrm{MC}$ \\
\hline 71 & Brassolis sophorae laurentii Stichel, 1925 & $\mathrm{~J}$ \\
\hline 72 & $\begin{array}{l}\text { Caligo illioneus illioneus (Cramer, 1775) } \\
\text { Satyrinae - Satyrini }\end{array}$ & $\mathrm{MC}$ \\
\hline 73 & Cissia terrestris (Butler, 1866) & CSS \\
\hline 74 & Euptychia westwoodi Butler, 1867 & $\mathrm{MC}$ \\
\hline 75 & Hermeuptychia hermes (Fabricius, 1775) & AS \\
\hline 76 & $\begin{array}{l}\text { Pareuptychia ocirrhoe ocirrhoe } \\
\text { (Fabricius, 1777) }\end{array}$ & $\mathrm{MC}$ \\
\hline 77 & $\begin{array}{l}\text { Paryphthimoides phronius } \\
\text { (Godart, [1824]) }\end{array}$ & $\mathrm{MC}$ \\
\hline 78 & Paryphthmoides poltys (Prittwitz, 1865) & $\mathrm{MC}$ \\
\hline 79 & Praefaunula armilla (Bulter, 1867) & CSS \\
\hline 80 & Yphthimoides ochracea (Butler, 1867) & AS \\
\hline 81 & $\begin{array}{l}\text { Yphthimoides yphthima } \\
\text { (C. Felder \& R. Felder, 1867) }\end{array}$ & AS \\
\hline 82 & $\begin{array}{l}\text { Yphthimoides celmis (Godart, [1824]) } \\
\text { Charaxinae - Anaeini }\end{array}$ & AS \\
\hline 83 & $\begin{array}{l}\text { Siderone galanthis (Cramer, 1775) } \\
\text { Biblidinae - Biblidini }\end{array}$ & CSS \\
\hline 84 & $\begin{array}{l}\text { Byblis hyperia nectanabis } \\
\text { (Fruhstorfer, 1909) }\end{array}$ & $\mathrm{MC}$ \\
\hline 85 & Eunica bechina (Hewitson, 1852) & CSS \\
\hline 86 & Eunica cuvierii (Godart, 1819) & CSS \\
\hline 87 & $\begin{array}{l}\text { Hamadryas laodamia laodamia } \\
\text { (Cramer, 1777) }\end{array}$ & $\mathrm{MC}$ \\
\hline 88 & $\begin{array}{l}\text { Hamadryas amphinome amphinome } \\
\text { (Linnaeus, 1767) }\end{array}$ & $\mathrm{MC}, \mathrm{CSS}$ \\
\hline 89 & Hamadryas februa (Hübner, [1823]) & CSS \\
\hline 90 & $\begin{array}{l}\text { Hamadryas feronia feronia } \\
\text { (Linnaeus, 1758) }\end{array}$ & CSS \\
\hline 91 & Temenis laothoe ssp. & CSS \\
\hline 92 & $\begin{array}{l}\text { Dynamine agacles agacles } \\
\text { (Dalman, 1823) }\end{array}$ & $\mathrm{MC}$ \\
\hline 93 & Callicore astarte selima (Guenée,1872) & CSS \\
\hline 94 & Callicore sorana sorana (Godart, [1824]) & CSS \\
\hline 95 & $\begin{array}{l}\text { Diaethria clymena janeira } \\
\text { (C. Felder, 1862) }\end{array}$ & $\mathrm{MC}$ \\
\hline
\end{tabular}

Tabela 1. Continuação...

\begin{tabular}{|c|c|c|}
\hline & Família/subfamília/tribo/espécies & Habitats \\
\hline & Nymphalinae - Coeini & \\
\hline 96 & Colobura dirce dirce (Linnaeus, 1758) & $\mathrm{MC}$ \\
\hline 97 & Historis odius dious Lamas, 1995 & CSS \\
\hline 98 & $\begin{array}{l}\text { Smyrna blomfildia blomfildia } \\
\text { (Fabricius, 1781) }\end{array}$ & $\mathrm{MC}$ \\
\hline \multirow[t]{2}{*}{99} & Tigridia acesta latifascia (Butler, 1873) & $\mathrm{MC}, \mathrm{J}$ \\
\hline & Nymphalinae - Nymphalini & \\
\hline 100 & Vanessa braziliensis (Moore, 1883) & $\mathrm{J}$ \\
\hline \multirow[t]{2}{*}{101} & Vanessa myrinna (Doubleday, 1849) & $\mathrm{CSS}, \mathrm{J}$ \\
\hline & Nymphalinae - Kallimini & \\
\hline 102 & Junonia evarete evarete (Cramer, 1779) & CSS \\
\hline 103 & $\begin{array}{l}\text { Anartia amathea roeselia } \\
\text { (Eschscholtz, 1821) }\end{array}$ & CSS, AS, J \\
\hline 104 & $\begin{array}{l}\text { Anartia jatrophae jatrophae } \\
\text { (Linnaeus, 1763) }\end{array}$ & CSS, AS, J \\
\hline \multirow[t]{2}{*}{105} & $\begin{array}{l}\text { Siproeta stelenes stelenes } \\
\text { (Linnaeus, 1758) }\end{array}$ & $\mathrm{MC}$ \\
\hline & Nymphalinae - Melitaeini & \\
\hline 106 & $\begin{array}{l}\text { Chlosyne lacinia saundersi } \\
\text { (Doubleday, [1847]) }\end{array}$ & $\mathrm{MC}, \mathrm{J}$ \\
\hline 107 & Eresia eunice esora Hewitson, 1857 & $\mathrm{MC}$ \\
\hline 108 & Eresia lansdorfi (Godart, 1819) & $\mathrm{MC}, \mathrm{J}$ \\
\hline 109 & Ortilia dicoma (Hewitson, 1864) & CSS \\
\hline 110 & Ortilia ithra (Kirby, 1900) & $\mathrm{J}$ \\
\hline 111 & Phystis simois simois (Hewitson, 1864) & CSS \\
\hline \multirow[t]{2}{*}{112} & Tegosa claudina (Eschscholtz, 1821) & $\mathrm{MC}, \mathrm{J}$ \\
\hline & Limenitidinae - Limenitidini & \\
\hline 113 & $\begin{array}{l}\text { Adelpha iphiclus ephesa } \\
\text { (Ménétriés, 1857) }\end{array}$ & CSS \\
\hline 114 & $\begin{array}{l}\text { Adelpha cytherea aea } \\
\text { (C. Felder \& R. Felder, 1867) }\end{array}$ & CSS \\
\hline 115 & Adelpha thoasa gerona (Hewitson, 1867) & CSS \\
\hline
\end{tabular}

CSS, AS

116 Euptoieta hegesia meridiania Stichel, 1938

Heliconiinae - Acraeini

117 Actinote discrepans d'Almeida 1958 MC, CSS

118 Actinote parapheles Jordan, 1913

119 Actinote pellenea pellenea

Hübner, [1821]

Heliconiinae - Heliconiini

120 Agraulis vanillae maculosa (Stichel, [1908])

121 Dione juno suffumata (Brown \& Mielke, 1972)

122 Dryadula phaetusa (Linnaeus, 1758)

CSS

MV, CSS

CSS, AS, J

CSS, MC, AS

CSS, J

$\mathrm{MC}, \mathrm{J}$

CSS, MC

124 Eueides isabella dianasa (Hübner, [1806])

125 Eueides aliphera aliphera (Godart, 1819) MC

126 Heliconius ethilla narcaea Godart, $1819 \quad \mathrm{MC}, \mathrm{J}$

127 Heliconius erato phyllis (Fabricius, 1775) MC, J

128 Heliconius sara thamar (Hübner, [1806]) MC, J 
$53,13 \%$ ) ocorreu neste tipo de vegetação, estando aí incluída a maioria dos Pieridae, Lycaenidae, Riodinidae e Nymphalidae (especialmente Biblidinae e Limenitidinae). Muitas dessas espécies (especialmente Pieridae e alguns Nymphalidae) também foram observadas em áreas abertas originalmente ocupadas por cerrado sensu stricto e atualmente apresentando diferentes estágios de sucessão ecológica. Outra parte considerável das espécies $(n=57$ ou 44,5\%) foi encontrada nas matas ciliares (especialmente Danainae, Ithomiinae, Morphinae e Heliconiinae), sendo que uma proporção menor dessas espécies também foi observada nos jardins do campus ( $\mathrm{n}=30$ ou 23,4\%). Alguns Papilionidae como Heraclides thoas brasiliensis, H. anchisiades capys e Battus polydamas polydamas foram observadas em todos os ambientes amostrados.

Além das espécies citadas na Tabela 1, podemos ainda esperar que novos registros de espécies sejam adicionados à nossa lista - uma relação de Papilionoidea que ocorrem em áreas próximas ao campus pode ser encontrada em Emery et al. (2006), Pinheiro (2006) e Pinheiro \& Emery (2006). Entre os grupos que esperamos obter novos registros estão os Lycaenidae, que contrariamente à maioria das borboletas são mais abundantes durante a estação seca (obs. pessoal) e contém muitas espécies com parte de seu ciclo de vida ocorrendo nos botões florais de várias plantas do cerrado, o que dificulta em muito a sua detecção pelos métodos de amostragem mais clássicos (Lewinsohn et al. 2005) e vários Riodinidae cuja biologia e plantas-hospedeiras só recentemente passaram a ser investigadas (Diniz \& Morais 1995, 1997, Diniz et al. 2001).

Várias borboletas típicas de matas ciliares, como Grasseia menelaus, Morpho helenor achillides (Morphinae), Caligo illioneus illioneus (a borboleta coruja; Brassolini), Lycorea halia discreta (Danainae), Byblis hyperia nectanabis, Hamadryas laodamia laodamia, Diaethria clymena janeira (Biblidinae) e Siproeta stelenes stelenes (Nymphalinae) foram raramente observadas, não parecendo constituir populações estáveis nestes locais. Outras espécies como Heliconius erato phyllis, $H$. sara thamar e H. ethilla narcaea (Heliconiinae) formam apenas pequenas populações na RCO e EEB, nem sempre presentes ao longo de todo o ano. Essa baixa riqueza de espécies e de indivíduos encontrada nas matas ciliares provavelmente ocorre em função de vários fatores, como a ausência ou raridade de plantas-hospedeiras, da pequena área que esse tipo de vegetação ocupa na região do campus e das condições ambientais que prevalecem nesse habitat, como um pequeno grau de sombreamento (grandes áreas sombreadas constituem uma condição necessária para o aparecimento de diversos grupos de borboletas, como os Charaxinae e vários Satyrinae, Nymphalinae, Papilionidae, Riodinidae e Lycaenidae, encontradas no Brasil central apenas em matas ciliares mais densas; Pinheiro \& Ortiz 1992; Pinheiro 2005) e baixa umidade relativa do ar, que afeta fortemente a ocorrência dos Ithomiinae, um grupo de borboletas que se abrigam nos locais mais sombreados e com maior umidade relativa durante o período da seca - maio a setembro (DeVries 1987, Brown Jr. 1992). Além desses fatores, a ocorrência de incêndios durante o período mais seco do ano provavelmente prejudica o aparecimento de um grande número de borboletas. Para exemplificar a forte influência que o fogo exerce sobre a comunidade de borboletas foi constatado que, seis meses após a ocorrência de um grande incêndio na mata da RCO, ocorrido em agosto de 2007, nenhuma das espécies comumente observadas naquele local durante a época de chuvas, como vários Ithomiinae e Heliconiinae, foi encontrada. De forma semelhante, várias espécies comumente encontradas nas áreas de cerrado do campus em 2004 e 2005, como Siderone galanthis, Colobura dirce dirce, Temenis laothoe, Junonia evarete evarete, Eunica bechina, E. cuvierii, Historis odius dious, Smyrna blomfildia blomfildia, Vanessa braziliensis e Vanessa myrinna não foram observadas nos levantamentos de fauna mais recentes (2006 a 2008). Esses resultados sugerem que a ocorrência de incêndios nas áreas de mata ciliar e cerrado sensu stricto do campus podem estar mesmo causando uma perda na riqueza de espécies de borboletas, restando no local apenas as espécies com maior capacidade de dispersão e colonização de novos habitats (Hill et al. 2001).

De fato a riqueza de espécies de borboletas no campus poderia ser bem maior caso algumas medidas fossem tomadas, como: 1) um maior controle sobre as queimadas que anualmente ocorrem em toda a região, com a formação de aceiro e a remoção de plantas invasoras como o capim, que favorecem a expansão do fogo entre as manchas de vegetação existentes; 2) o estabelecimento de áreas visando à regeneração da vegetação original de cerrado; 3) a introdução de plantas típicas do bioma cerrado em várias localidades onde a vegetação original foi quase totalmente removida e, especialmente, junto às pequenas manchas de mata ciliar na RCO, EEB e Arboreto - aumentando a área atual ocupada por matas no campus; 4) a remoção do lixo e dos animais domésticos presentes; e 5) um maior controle na entrada e no fluxo de pessoas. Estas medidas certamente aumentariam não apenas o número de borboletas, mas de toda a flora e fauna presente no campus.

\section{Agradecimentos}

Somos gratos a Keith S., Brown Jr., André V. L. Freitas, Paulo C. Motta e Mirma M. Casagrande pela colaboração na identificação de espécies ocorrida ao longo dos últimos anos e pela enriquecedora troca de idéias.

\section{Referências Bibliográficas}

BROWN Jr., K.S. 1992. Borboletas da Serra do Japi: diversidade, habitats, recursos alimentares e variação temporal. In Historia Natural da Serra do Japi: ecologia e preservação de uma área florestal no sudeste do Brasil (L. Morellato, org.). Unicamp, Campinas, p. 142-187.

BROWN Jr., K.S. \& GIFFORD, D.R. 2002. Lepidoptera in the cerrado landscape and the conservation of vegetation, soil, and topographical mosaics. In The cerrados of Brazil: ecology and natural history of a neotropical savanna (P.S. Oliveira \& R.J. Marquis eds.). Columbia Univ. Press, New York, p. 201-217.

BROWN Jr., K.S. \& MIELKE, O.H.H. 1967a. Lepidoptera of the Central Brazil Plateau. I. Preliminary list of Rhopalocera: Introduction, Nymphalidae, Libytheidae. J. Lepid. Soc. 21(2):77-106.

BROWN Jr., K.S. \& MIELKE, O.H.H. 1967b. Lepidoptera of the Central Brazil Plateau. II. Preliminary list of Rhopalocera (continued): Lycaenidae, Pieridae, Papilionidae, Hesperiidae. J. Lepid. Soc. 21(3):145-168.

DEVRIES, P.J. 1987. The butterflies of Costa Rica and their natural history. Vol. 1. Papilionidae, Pieridae and Nymphalidae. Princeton Univ. Press, Princeton, p. 1-327.

DEVRIES, P.J., WALLA, T.R. \& GREENEY, H.F. 1999. Species diversity in spatial and temporal dimensions of fruity feeding butterflies from two Ecuadorian rainforests. Biol. J. Linn. Soc. 68(3):333-353.

DINIZ, I.R. \& MORAIS, H.C. 1995. Larvas de Lepidoptera e suas plantas hospedeiras em um cerrado de Brasília, Distrito Federal, Brasil. Rev. Bras. Entom. 39(2):755-770.

DINIZ, I.R. \& MORAIS, H.C. 1997. Lepidopteran caterpillar fauna of cerrado host plants. Biodiv. and Conserv. 6:817-836.

DINIZ, I.R., MORAIS, H.C. \& CAMARGO, A.J.A. 2001. Host plants of lepidopteran caterpillars in the cerrado of the Distrito Federal. Rev. Bras. Entom. 45(2):107-122.

EITEN, G. 1972. The cerrado vegetation of central Brazil. Bot. Rev. 38(2):205-341.

EMERY, E.O., BROWN Jr., K.S. \& PINHEIRO, C.E.G. 2006. As borboletas (Lepidoptera, Papilionoidea) do Distrito Federal. Rev. Bras. Entom. 50(1):85-92. 
GOODLAND, R. 1971. A physiognomic analysis of the cerrado vegetation of central Brazil. J. Ecol. 59(2):411-419.

HILL, J.K., HAMER, K.C., TANGAH, J. \& DAWOOD, M. 2001. Ecology of tropical butterflies in forest gaps. Oecologia 128(2):294-302.

LAMAS, G. 2004. Checklist: Part 4A. Hesperioidea - Papilionoidea. In Atlas of Neotropical Lepidoptera. Volume 5A. (J.B. Heppner, ed.). Association for Tropical Lepidoptera, Inc. Scientific Publ., Gainesville.

LEWINSOHN, T.M., FREITAS, A.V.L. \& PRADO, P.I. 2005. Conservation of terrestrial invertebrades and their habitats in Brazil. Cons. Biol. 19(3):640-645.

MYERS, N., MITTERMEIER, R.A., MITTERMEIER, C.G., FONSECA, G.A.B. \& KENT, J. 2000. Biodiversity hotspots for conservation priorities. Nature 403:853-858.

OLIVEIRA-FILHO, A.T. \& RATTER, J. 2002. Vegetation physiognomies and woody flora of the cerrado biome. In The cerrados of Brazil: ecology and natural history of a neotropical savanna (Oliveira, P.S. \& Marquis, R.J. eds.). Columbia Univ. Press, New York, p. 91-120.

Pinheiro, C.E.G. 2005. Estudos comparativos sobre a fauna de borboletas do Distrito Federal: Implicações para a conservação. In Biodiversidade,
Ecologia e Conservação do Cerrado (Scariot, A., Silva, J.C.S. \& Felfili, J.M. eds.). MMA, Brasília, p. 297-303.

PINHEIRO, C.E.G. 2006. A fauna de borboletas (Insecta, Lepidoptera) da APA de Cafuringa. In Apa de Cafuringa, a última fronteira natural do DF (Netto, P.B., Mecenas, V.V. \& Cardoso, E.S., eds.). Semarh, Brasília, p. 231-234.

PINHEIRO, C.E.G. \& EMERY, E.O. 2006. As borboletas (Lepidoptera: Papilionoidea e Hesperioidea) da Área de Proteção Ambiental do Gama e Cabeça de Veado (Distrito Federal, Brasil). Biota Neotrop. 6(3):http:// www.biotaneotropica.org.br/v6n3/pt/abstract?inventory+bn0150603200 6 (último acesso em 17/05/2008).

PINHEIRO, C.E.G. \& ORTIZ, J.V.C. 1992. Communities of fruit-feeding butterflies along a vegetation gradient in central Brazil. J. Biogeog. 19:505-511

Recebido em 15/05/08 Versão reformulada recebida em 30/09/08 Publicado em 09/10/08 